\title{
Topological magnon bands and unconventional thermal Hall effect on the frustrated honeycomb and bilayer triangular lattice* ${ }^{*}$
}

\author{
S. A. Owerre ${ }^{1}$ \\ ${ }^{1}$ Perimeter Institute for Theoretical Physics, 31 Caroline St. N., Waterloo, Ontario N2L 2Y5, Canada.
}

(Dated: November 9, 2018)

\begin{abstract}
In the conventional ferromagnetic systems, topological magnon bands and thermal Hall effect are due to the Dzyaloshinskii-Moriya interaction (DMI). In principle, however, the DMI is either negligible or it is not allowed by symmetry in some quantum magnets. Therefore, we expect that topological magnon features will not be present in those systems. In addition, quantum magnets on the triangular-lattice are not expected to possess topological features as the DMI or spin-chirality cancels out due to equal and opposite contributions from adjacent triangles. Here, however, we predict that the isomorphic frustrated honeycomb-lattice and bilayer triangular-lattice antiferromagnetic system will exhibit topological magnon bands and topological thermal Hall effect in the absence of an intrinsic DMI. These unconventional topological magnon features are present as a result of magnetic-field-induced non-coplanar spin configurations with nonzero scalar spin chirality. The relevance of the results to realistic bilayer triangular antiferromagnetic materials are discussed.
\end{abstract}

\section{INTRODUCTION}

In recent years, the concept of topological band theory has been extended to bosonic systems. Consequently, topological magnon bands and the associated thermal Hall effect in insulating quantum ferromagnets have garnered considerable attention. The experimental realizations of these phenomena in the quasi-two-dimensional (2D) kagomé ferromagnets $\mathrm{Cu}(1-3, \mathrm{bdc})^{1 / 2}$ have further rekindled much interest in this area. Thermal Hall effect of magnons was previously realized experimentally in different $3 \mathrm{D}$ pyrochlore ferromagnets $\mathrm{A}_{2} \mathrm{~B}_{2} \mathrm{O}_{7} \stackrel{34}{\text {. }}$. These experimental studies follow from different theoretical proposals 5 . An extension to unfrustrated honeycomb magnets has been recently proposed by different authors 14$]$.

Generally speaking, it is believed that the topological magnon phenomena in quantum ferromagnets 14 result from the $\mathrm{DMl}^{2021}$, which plays the same role as spinorbit coupling (SOC) in electronic systems 2223 . To date, there is no experimental evidence of a counter-example where topological magnon features originate from an alternative source other than the DMI in any magnetically ordered system. Therefore, the conception is that the DMI is mandatory for topological magnon properties to exist in magnetically ordered systems.

The frustrated magnets provide a platform to explore this possibility as we have previously shown on the kagomé-type lattices 2425. But the kagomé-type lattices naturally allow an intrinsic DMI, which is capable of inducing and stabilizing the coplanar spin structure ${ }^{26}$. In contrast, most non-kagomé-type frustrated magnets do not allow an intrinsic DMI due to symmetry. Therefore, we wish to extend our analyses to those systems. On the honeycomb lattice, geometric frustration is present when a next-nearest-neighbour (NNN) antiferromagnetic interaction $\mathcal{J}_{2}$ competes with a nearest-neighbour (NN) antiferromagnetic interaction $\mathcal{J}_{1}$. This system is known as the frustrated $\mathcal{J}_{1}-\mathcal{J}_{2}$ honeycomb-lattice Heisenberg anti- ferromagnet (HLHAF). It possesses interesting phase diagram for $\mathcal{J}_{2} / \mathcal{J}_{1} \ll 1^{27}+40$. The opposite limit $\mathcal{J}_{2} / \mathcal{J}_{1} \gg 1$ is unexplored. In this regime the geometric frustration induced by $\mathcal{J}_{2}$ yields a decoupled $120^{\circ}$ coplanar order. The model is now isomorphic to a $120^{\circ}$ coplanar order on the stacked bilayer triangular-lattice Heisenberg antiferromagnet (TLHAF) with intraplane coupling $\mathcal{J}_{2}$ and small interplane coupling $\mathcal{J}_{1} \underline{41}$. Therefore, one can capture the physics of bilayer TLHAF by studying the dominant $\mathcal{J}_{2}$ limit of the frustrated HLHAF.

In this paper, we predict that topological magnon bands and unconventional topological thermal Hall effect will exist on the isomorphic honeycomb and bilayer triangular lattice $\mathcal{J}_{1}-\mathcal{J}_{2}$ model without any DMI. We show that the system possesses topological magnon bands with nonzero Chern number $\mathcal{C}_{ \pm}= \pm \operatorname{sgn}(\sin \phi)$, where $\sin \phi$ is related to the field-induced scalar spin chirality $\chi=\sum \mathcal{S}_{i} \cdot\left(\mathcal{S}_{j} \times \mathcal{S}_{k}\right)$, and $i, j, k$ label sites on a unit triangle; $\phi$ is the angle subtended by three non-coplanar (umbrella) spins. The corresponding thermal Hall conductivity $\kappa_{x y}$ is tunable by the external magnetic field as it requires no DMI. Interestingly, topological properties are not expected to be present on the triangular lattice as the DMI (spin-chirality) cancels out due to equal and opposite contributions from adjacent triangles 5 . The current result is a counter-example where the field-induced scalar-chirality of the non-coplanar (umbrella) spin structure does not cancel as it is coupled to the magnetization of the non-coplanar spins 42 .

These results are particularly interesting especially for the stacked triangular-lattice antiferromagnetic materials with no intrinsic DMI. They include $\mathrm{Ba}_{3} \mathrm{XSb}_{2} \mathrm{O}_{9}(\mathrm{X}$ $\equiv \mathrm{Mn}, \mathrm{Co}$, and $\mathrm{Ni})^{43}-49$ and $\mathrm{VX}_{2}(\mathrm{X} \equiv \mathrm{Cl}, \mathrm{Br}$, and I) and others 52 . The effects of topological magnons are also manifested by the measurement of nonzero thermal Hall conductivity $\kappa_{x y}$ at various external magnetic fields along the $\hat{z}$-axis ${ }^{12}$. We note that the quasi-2D bilayer metallic triangular-lattice magnet $\mathrm{PdCrO}_{2}$ with $120^{\circ}$ coplanar order also shows a finite anomalous Hall effect in 
a perpendicular-to-plane external magnetic field 53 . We therefore expect that an analogous thermal Hall effect in magnetic insulators with charge-neutral excitations such as magnons will be present and of great importance.

\section{MODEL}

The frustrated isomorphic $\mathcal{J}_{1}-\mathcal{J}_{2}$ Heisenberg model on the honeycomb- and bilayer triangular-lattice in an external magnetic field is given by

$$
\mathcal{H}=\sum_{i j} \mathcal{J}_{i j} \mathcal{S}_{i} \cdot \mathcal{S}_{j}-H \sum_{i} \mathcal{S}_{i}^{z}
$$

where $\mathcal{J}_{i j}=\mathcal{J}_{1}\left(\mathcal{J}_{2}\right)$ are nearest (next-nearest) neighbour antiferromagnetic interactions and $H$ is the external magnetic field along the $z$-axis perpendicular to the lattice plane. The Hamiltonian (1) has been extensively studied on the honeycomb lattice in the context of ground state (thermodynamic) properties $28 \mid 40$. In the classical limit at zero magnetic field $\frac{28 \mid 29}{}$, a collinear Néel order exists for $\mathcal{J}_{2} / \mathcal{J}_{1}<1 / 6$. For $\mathcal{J}_{2} / \mathcal{J}_{1}>1 / 6$ it has a family of degenerate spiral order with incommensurate wave vectors. It was shown that spin wave fluctuations at leading order lift this accidental degeneracy in favour of specific wave vectors. In particular, the $120^{\circ}$ coplanar order with ordering wave vector $\mathbf{K}=\mathbf{Q}=( \pm 2 \pi / 3 \sqrt{3}, 2 \pi / 3)$ is expected to emerge for $\mathcal{J}_{2} / \mathcal{J}_{1} \gg 1$. In this limit the Hamiltonian (1) is isomorphic to the bilayer TLHAF with intraplane coupling $\mathcal{J}_{2}$ and small interplane coupling $\mathcal{J}_{1}$. The two sublattices of the honeycomb lattice $A, B$ are equivalent to the top and bottom sublattices of the bilayer triangular lattice as shown in figure (1). Therefore, we study both systems simultaneously via equation (1).

\section{RESULTS}

\section{A. Magnon band structures}

It is advantageous to introduce the standard HolsteinPrimakoff bosonization. The calculation is tedious but doable as shown in Appendix A. We have checked that for $\mathcal{J}_{2} / \mathcal{J}_{1}<1 / 6$ or equivalently $\mathcal{J}_{2} / \mathcal{J}_{1} \rightarrow 0$ the Hamiltonian recovers the magnon band structures of collinear (canted) Néel antiferromagnet at $H=0\left(H<H_{s}\right)$ as well as collinear ferromagnet at $H=H_{s}$, where $H_{s}=3\left(2 \mathcal{J}_{1}+3 \mathcal{J}_{2}\right)$ is the saturation field. These limiting cases require the DMI for topological features to exist as previously shown $14-19$.

We are interested in the dominant $\mathcal{J}_{2}$ limit corresponding to the isomorphic HLHAF and bilayer TLHAF with a stable $120^{\circ}$ coplanar order. In this regime the magnetic field induces a non-coplanar (umbrella) chiral spin texture with nonzero scalar spin chirality $\chi$. We have shown the magnon bands at zero magnetic field $H=0$ in figure (2) (i.e., conventional $120^{\circ}$ spin structure). They have
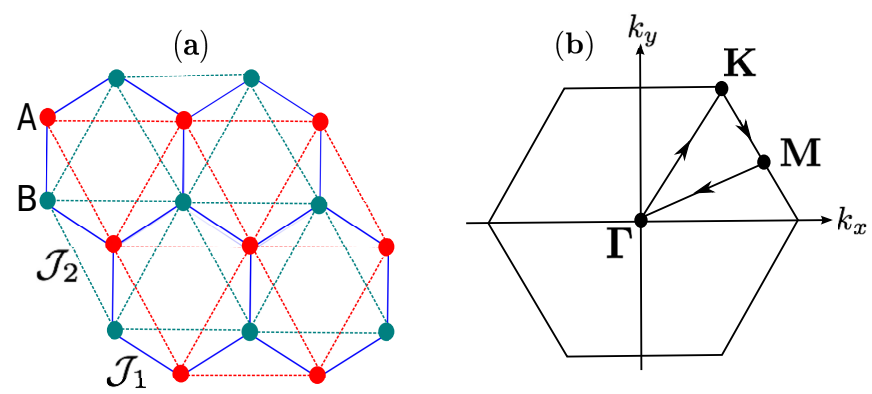

FIG. 1: Color online. (a) Schematics of the isomorphic $\mathcal{J}_{1}-\mathcal{J}_{2}$ honeycomb-lattice and bilayer triangular-lattice. (b) The first Brillouin zone of the system with indicated paths.

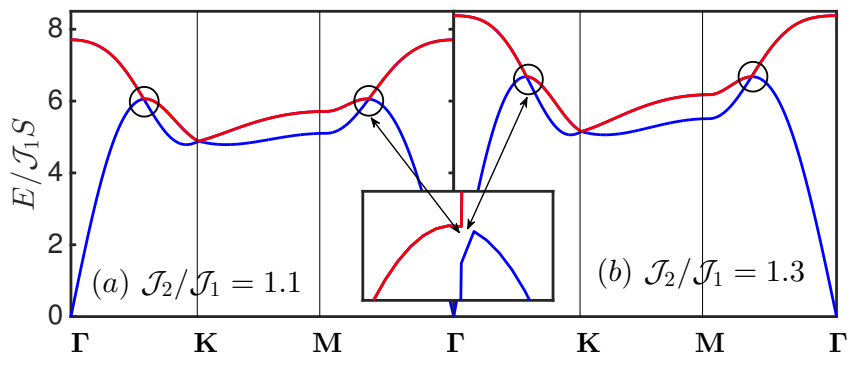

FIG. 2: Color online. Dirac magnon bands of the conventional $120^{\circ}$ coplanar order at zero magnetic field $H=0$. The bands linearly touching at $\mathbf{K}$ and form a Dirac point. Inset shows the circled points

Dirac point nodes at $\mathbf{K}$. The Dirac nodes remain intact even in the presence of (out-of-plane) DMI as it plays a stability role in certain frustrated magnets rather than a topological role. In figure (3) we have shown the magnon bands for $\mathcal{J}_{2} / \mathcal{J}_{1}=1.3$ at two values of nonzero magnetic fields. We see that the Dirac magnon nodes are gapped and the magnon bands become topological due to the presence of nonzero scalar spin chirality $\chi$. Notice that there is a roton-minimum near the ordering wave vector of the coplanar spin structure at $\mathbf{K}=\mathbf{Q}$, which becomes gapless for large $\mathcal{J}_{2} / \mathcal{J}_{1}$. These features can be reproduced in the bilayer triangular-lattice antiferromagnetic systems as shown explicitly in Appendix (B).

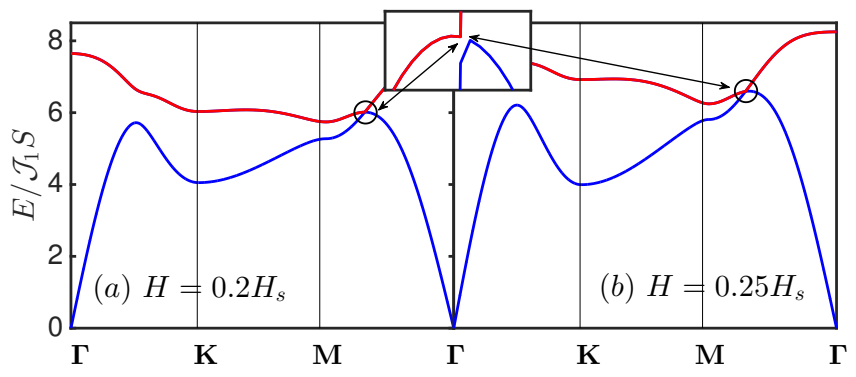

FIG. 3: Color online. Topological magnon bands of the noncoplanar (umbrella) spin structure for $\mathcal{J}_{2} / \mathcal{J}_{1}=1.3$ at two magnetic field values. Inset shows the circled points. 


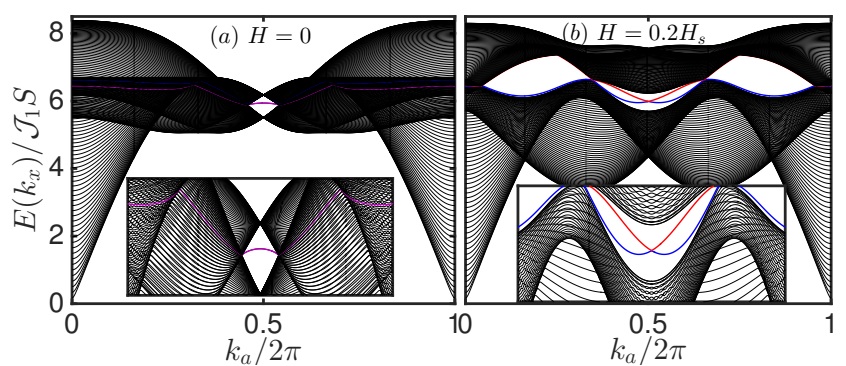

FIG. 4: Color online. Magnon chiral edge modes for a strip geometry periodic in $x$, but with open boundaries along $y$ for $\mathcal{J}_{2} / \mathcal{J}_{1}=1.3$. The black region corresponds to the magnon bulk bands. The chiral edge modes are shown in colors. (a) Dirac cones connected by flat chiral edge modes at zero magnetic field $H=0$. (b) Gapped Dirac cones with gapless chiral edge modes for small magnetic field $H=0.2 H_{s}$ as a result of the emergent scalar spin chirality $\chi$. Insets show the magnified chiral edge modes.

\section{B. Berry curvature and Chern number}

Most importantly, the magnon bands with nonzero scalar spin chirality $\chi$ now acquire a nonzero Berry curvature, given by

$$
\Omega_{i j ; \mathbf{k} s}=-\sum_{s \neq s^{\prime}} \frac{2 \operatorname{Im}\left[\left\langle\mathcal{P}_{\mathbf{k} s}\left|v_{i}\right| \mathcal{P}_{\mathbf{k} s^{\prime}}\right\rangle\left\langle\mathcal{P}_{\mathbf{k} s^{\prime}}\left|v_{j}\right| \mathcal{P}_{\mathbf{k} s}\right\rangle\right]}{\left(E_{\mathbf{k} s}-E_{\mathbf{k} s^{\prime}}\right)^{2}}
$$

where $v_{i}=\partial\left(\eta \mathcal{H}_{\mathbf{k}}\right) / \partial k_{i}$ defines the velocity operators and $\eta=\operatorname{diag}\left(I_{N \times N},-I_{N \times N}\right)$ is the diagonal of $N \times N$ identity matrix and $s$ labels the bands. Here $\mathcal{P}_{\mathbf{k} s}$ is the paraunitary operator that diagonalizes $\eta \mathcal{H}_{\mathbf{k}}$. The Chern numbers are given by

$$
\mathcal{C}_{s}=\frac{1}{2 \pi} \int_{B Z} d k_{i} d k_{j} \Omega_{i j ; \mathbf{k} s}
$$

We have computed the Chern numbers numerically and established that for the two positive magnon bands, say $s= \pm, \mathcal{C}_{ \pm}= \pm \operatorname{sgn}(\sin \phi)$, where $\sin \phi$ is related to the field-induced scalar spin chirality $\chi$ and $\phi$ is the angle subtended by three non-coplanar (umbrella) spins. It changes sign by reversing the sign of the magnetic field or the scalar spin chirality, i.e., $\sin \phi \rightarrow-\sin \phi$ as $\vartheta \rightarrow \vartheta+\pi$.

The existence of chiral magnon edge modes is another aspect of topological character of nontrivial magnons in insulating quantum magnets. At zero magnetic field the Dirac magnon bulk bands are connected by a flat chiral edge mode as shown in figure (4) (a). As the magnetic field is turned on the flat edge modes are lifted due the presence of scalar spin chirality $\chi$ as shown in figure (4) (b). The chiral edge modes are now topologically protected by the Chern numbers $\mathcal{C}_{ \pm}= \pm \operatorname{sgn}(\sin \phi)$.
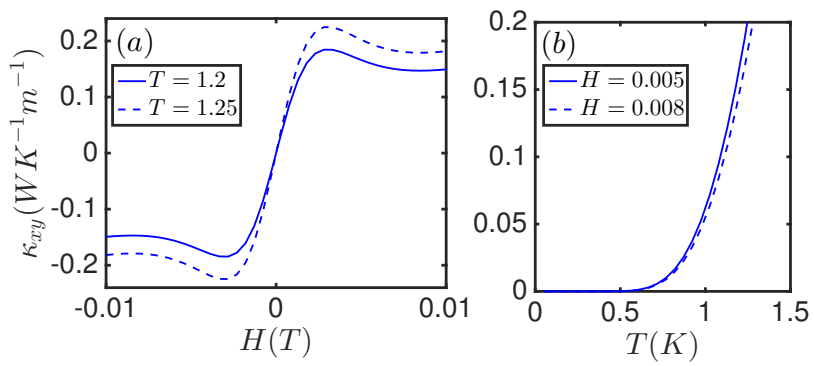

FIG. 5: Color online. (a) Tunable thermal Hall conductivity $\kappa_{x y}$ as a function of magnetic field $H$ for two temperature values. (b) Tunable thermal Hall conductivity $\kappa_{x y}$ as a function of temperature $T$ for two magnetic field values. The coupling is set to $\mathcal{J}_{2} / \mathcal{J}_{1}=1.3$.

\section{Topological thermal Hall effect}

Conventionally, thermal Hall effect is induced by the DMI as reported in unfrustrated magnets ${ }^{1 / 19}$ as well as frustrated magnets $54 \sqrt[56]{5}$. For frustrated magnets with noncollinear and coplanar spin structure thermal Hall conductivity can be nonzero in the absence of the DMI. This is possible because an external magnetic field can induce non-coplanar spin configurations with nonzero scalar spin chirality $\chi$. The interesting feature in the current study is that the coplanar spin structure can be present in bilayer TLHAF without an intrinsic DMI 43 . The thermal Hall conductivity $\kappa_{x y}$ can be derived from linear response theory 10 . We have shown the trends of $\kappa_{x y}$ as functions of the magnetic field and temperature in figures (5) (a) and (b) respectively for a specific value of $\mathcal{J}_{2} / \mathcal{J}_{1}$. Evidently, we capture a sign change in $\kappa_{x y}$ as the scalar spin chirality is reversed by reversing the sign of the magnetic field. Interestingly, the trend of $\kappa_{x y}$ is synthetic and tunable by the magnetic field as it requires no intrinsic DMI. As we discuss below certain (honeycomb) triangular-lattice antiferromagnetic materials do not have an intrinsic DMI, so we do expect that $\kappa_{x y}$ can be tuned by an external magnetic field.

\section{CONCLUSION}

We have predicted that topological magnon bands and unconventional topological thermal Hall effect will exist in the isomorphic $\mathcal{J}_{1}-\mathcal{J}_{2}$ honeycomb-lattice and bilayer triangular-lattice antiferromagnets. These interesting features originate from the magnetic-field-induced noncoplanar (umbrella) spin structure with nonzero scalar spin chirality and require no DMI in contrast to previously studied unfrustrated magnets 19 and frustrated magnets 54 57. Therefore they are unconventional and synthetic as they can be tuned by the external magnetic field.

Most importantly, topological magnon features have not been previously predicted on the triangular lattice 
for the reasons we mentioned above. In realistic materials the bilayer triangular antiferromagnetic systems do not usually allow an intrinsic DM interaction, but an easyplane (axis) anisotropy can be present. This is the case in the bilayer triangular-lattice quantum antiferromagnets $\mathrm{Ba}_{3} \mathrm{XSb}_{2} \mathrm{O}_{9}(\mathrm{X} \equiv \mathrm{Mn}$ and $\mathrm{Co})$ with a stable $120^{\circ}$ coplanar order and no intrinsic DM interaction 43 . They are also quasi-two-dimensional (quasi-2D) with dominant intraplane $\left(\mathcal{J}_{2}\right)$ coupling and small interplane coupling $\left(\mathcal{J}_{1}\right)$ and easy-axis anisotropy $(\Delta)$.

The compounds $\mathrm{VX}_{2}(\mathrm{X} \equiv \mathrm{Cl}, \mathrm{Br}$, and $\mathrm{I})$ also form quasi-2D layered triangular-lattice quantum antiferromagnets with a stable $120^{\circ}$ coplanar order and no in-

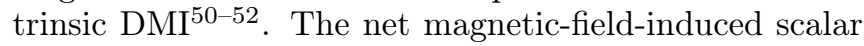
spin chirality in the non-coplanar regime will be nonzero. Therefore, the current predictions can be tested in these materials. Indeed, a finite $\kappa_{x y}$ at various external magnetic fields signifies that the magnetic excitations are topologically nontrivial. As mentioned above, we have previously shown slightly similar results on the kagomé and star lattices2425, but these lattice geometries naturally allow a DMI which stabilizes the coplanar spin structure $\sqrt{26}$. In contrast, the current results are different in that the bilayer triangular antiferromagnetic materials mentioned above do not have an intrinsic DMI and a non-vanishing spin-chirality can be induced by applying an external magnetic field in the plane perpendicular to the magnets.

The bilayer honeycomb-lattice quantum antiferromagnets $\mathrm{Bi}_{3} \mathrm{X}_{4} \mathrm{O}_{12}\left(\mathrm{NO}_{3}\right)(\mathrm{X} \equiv \mathrm{Mn}, \mathrm{V}$, and $\mathrm{Cr}$ ) are also promising candidates; however it is believed that $\mathcal{J}_{2} / \mathcal{J}_{1} \ll 1$ for $\mathrm{X} \equiv \mathrm{Mn}$, but an external magnetic field induces a transition to a 3D collinear Néel order at $H \sim 6 T^{27}$. In the collinear regime the DMI is mandatory for topological magnons to exist, and it can be allowed in this compound 58 . There is also a possibility to synthesize different honeycomb materials with dominant $\mathcal{J}_{2} \frac{59}{\text {. }}$ We also expect the spontaneously-induced spin chirality in the chiral spin liquid to have the same topological effects on the underlying magnetic excitations. Hence, the scalar-chirality mechanism can help explain the recently observed thermal Hall conductivity in a spin liquid material at nonzero magnetic field $\underline{60}$.

\section{Acknowledgements}

Research at Perimeter Institute is supported by the Government of Canada through Industry Canada and by the Province of Ontario through the Ministry of Research and Innovation.

\section{Appendix A: Dominant $\mathcal{J}_{2}$ limit}

We are interested in the dominant $\mathcal{J}_{2}$ limit of equation (1), corresponding to the isomorphic honeycomb-lattice and bilayer triangular-lattice antiferromagnets with a stable $120^{\circ}$ coplanar order. At zero field we take the spins to lie on the plane of the honeycomb (bilayer) triangle lattice taken as the $x y$ plane. Then, we perform a rotation about the $z$-axis on the sublattices by the spin oriented angles $\theta_{i}$. As the external magnetic field is turned on, the spins will cant towards the direction of the field and form a non-coplanar configuration. Thus, we have to align them along the new quantization axis by performing a rotation about the $y$-axis by the field canting angle $\vartheta$. The total transformation of the spins is

$$
\mathcal{S}_{i}=\mathcal{R}_{z}\left(\theta_{i}\right) \cdot \mathcal{R}_{y}(\vartheta) \cdot \mathcal{S}_{i}^{\prime}
$$

where

$$
\mathcal{R}_{z}\left(\theta_{i}\right) \cdot \mathcal{R}_{y}(\vartheta)=\left(\begin{array}{ccc}
\cos \theta_{i} \cos \vartheta & -\sin \theta_{i} & \cos \theta_{i} \sin \vartheta \\
\sin \theta_{i} \cos \vartheta & \cos \theta_{i} & \sin \theta_{i} \sin \vartheta \\
-\sin \vartheta & 0 & \cos \vartheta
\end{array}\right) .
$$

Next, we plug the spin transformation A1 into the Hamiltonian (1). There are numerous terms but we retain only the terms that contribute to the free magnon model, given by

$$
\begin{aligned}
\mathcal{H} & =\sum_{i j} \mathcal{J}_{i j}\left[\cos \theta_{i j} \mathcal{S}_{i}^{\prime} \cdot \mathcal{S}_{j}^{\prime}+\sin \theta_{i j} \cos \vartheta \hat{\mathbf{z}} \cdot\left(\mathcal{S}_{i}^{\prime} \times \mathcal{S}_{j}^{\prime}\right)\right. \\
& \left.+2 \sin ^{2}\left(\frac{\theta_{i j}}{2}\right)\left(\sin ^{2} \vartheta \mathcal{S}_{i}^{\prime x} \mathcal{S}_{j}^{\prime x}+\cos ^{2} \vartheta \mathcal{S}_{i}^{\prime z} \mathcal{S}_{j}^{\prime z}\right)\right] \\
& -H \cos \vartheta \sum_{i} \mathcal{S}_{i}^{\prime z}
\end{aligned}
$$

where $\theta_{i j}=\theta_{i}-\theta_{j}$. We note that for $\mathcal{J}_{i j}=\mathcal{J}_{1}$, $\sin \theta_{i j}=0$, therefore the field-induced scalar spin chirality of the non-coplanar (umbrella) spin configurations defined as $\chi=\sum \mathcal{S}_{i}^{\prime} \cdot\left(\mathcal{S}_{j}^{\prime} \times \mathcal{S}_{k}^{\prime}\right)$ is induced only within the triangular plaquettes of the NNN bonds on the honeycomb lattice or the triangular plaquettes of the NN bilayer triangular lattice. Here $\sin \theta_{i j}=\nu_{i j}\left|\sin \theta_{i j}\right|$, where $\nu_{i j}= \pm 1$ denotes the sign of the magnon hopping along the triangular plaquettes of the honeycomb (bilayer triangular) lattice.

Usually, the net chirality vanishes on the triangular lattice because neighbouring triangular plaquettes contribute equal and opposite chirality ${ }^{5}$. But the fieldinduced spin chirality of the non-coplanar (umbrella) spin structure will be finite as it is coupled to the magnetization of the non-coplanar spin configuration. The sign of the scalar-chirality is determined by the magnetic field and it has the same sign on each honeycomb (bilayer) triangle for $H>0$, whereas for $H<0$ the spins on each honeycomb (bilayer) triangle flip, now $\vartheta \rightarrow \pi+\vartheta$ on each triangle. In this case the net scalar-chirality is nonzerd 42 . The origin of the spin chirality can also be inferred from geometric frustration of the lattice, which can allow a chiral spin liquid phase. In this case, the scalar spin chirality can be spontaneously developed. Because of the scalar 
spin chirality the system has already acquired a real space Berry curvature from the chiral magnetic spin structure. We therefore expect the spontaneously-induced and the field-induced spin chirality to have the same topological effects on the underlying magnetic excitations.

In the present case, it is advantageous to introduce the Holstein-Primakoff bosonization $\frac{62 !}{} \mathcal{S}_{i}^{z}=S-a_{i}^{\dagger} a_{i}, \mathcal{S}_{i}^{+} \approx$ $\sqrt{2 S} a_{i}=\left(\mathcal{S}_{i}^{-}\right)^{\dagger}$, where $\mathcal{S}_{i}^{ \pm}=\mathcal{S}_{i}^{x} \pm i \mathcal{S}_{i}^{y}$ and $a_{i}^{\dagger}\left(a_{i}\right)$ are the bosonic creation (annihilation) operators. The magnon tight binding Hamiltonian is given by

$$
\begin{aligned}
\mathcal{H}_{\mathcal{J}_{1}} & =S \sum_{\langle i, j\rangle}\left[t_{1, z}\left(a_{i}^{\dagger} a_{i}+a_{j}^{\dagger} a_{j}\right)+t_{1, r}\left(a_{i}^{\dagger} a_{j}+\text { h.c. }\right) \quad(\mathrm{A} 4)\right. \\
& \left.+t_{1, o}\left(a_{i}^{\dagger} a_{j}^{\dagger}+h . c .\right)\right], \\
\mathcal{H}_{\mathcal{J}_{2}} & =S \sum_{\langle\langle i, j\rangle\rangle}\left[t_{2, z}\left(a_{i}^{\dagger} a_{i}+a_{j}^{\dagger} a_{j}\right)+t_{2}\left(e^{-i \phi_{i j}} a_{i}^{\dagger} a_{j}+\text { h.c. }\right)\right. \\
& \left.+t_{2, o}\left(a_{i}^{\dagger} a_{j}^{\dagger}+\text { h.c. }\right)\right] ; \mathcal{H}_{H}=H_{\vartheta} \sum_{i} a_{i}^{\dagger} a_{i},
\end{aligned}
$$

where $\langle i, j\rangle$ and $\langle\langle i, j\rangle\rangle$ denote the summations over the $\mathrm{NN}$ and NNN sites respectively.

$$
\begin{aligned}
& \left.t_{1, z}=\mathcal{J}_{1}\left[1-2 \cos ^{2} \vartheta\right)\right], t_{1, r}=-\mathcal{J}_{1}\left[1-\sin ^{2} \vartheta\right], \\
& t_{1, o}=\mathcal{J}_{1} \sin ^{2} \vartheta, t_{2, z}=\frac{\mathcal{J}_{2}}{2}\left[1-3 \cos ^{2} \vartheta\right], \\
& t_{2}=\sqrt{\left(t_{2, r}\right)^{2}+\left(t_{2, m}\right)^{2}}, t_{2, r}=-\frac{\mathcal{J}_{2}}{2}\left(1-3 \sin ^{2} \vartheta / 2\right) \\
& t_{2, m}=\frac{\sqrt{3} \mathcal{J}_{2}}{2} \cos \vartheta, t_{2, o}=\frac{3 \mathcal{J}_{2}}{4} \sin ^{2} \vartheta
\end{aligned}
$$

and $H_{\vartheta}=H \cos \vartheta$. The angle $\vartheta$ is determined from the mean-field energy, given by

$E_{0}=-\frac{3 \mathcal{J}_{1}}{2}\left(1-2 \cos ^{2} \vartheta\right)-\frac{3}{2} \mathcal{J}_{2}\left(1-3 \cos ^{2} \vartheta\right)-H \cos \vartheta$,

where $E_{0}=E_{M F} / N S^{2}$ and $N$ is the total number of sites on the honeycomb lattice. The magnetic field is rescaled in unit of $S$. Minimizing this energy yields the canting angle $\cos \vartheta=H / H_{s}$, where $H_{s}=3\left(2 \mathcal{J}_{1}+3 \mathcal{J}_{2}\right)$ is the saturation field. The solid angle subtended by three non-coplanar spins is given by $\phi_{i j}=\nu_{i j} \phi$, where $\phi=$ $\tan ^{-1}\left[t_{2, m} / t_{2, r}\right]$. In Fourier space the Hamiltonian can be written as $\mathcal{H}=\frac{1}{2} S \sum_{\mathbf{k}} \Psi_{\mathbf{k}}^{\dagger} \mathcal{H}_{\mathbf{k}} \Psi_{\mathbf{k}}+$ const., where $\Psi_{\mathbf{k}}=$ $\left(\psi_{\mathbf{k}}^{\dagger}, \psi_{-\mathbf{k}}\right)$, with $\psi_{\mathbf{k}}^{\dagger}=\left(a_{\mathbf{k}, A}^{\dagger}, a_{\mathbf{k}, B}^{\dagger}\right)$.

$$
\mathcal{H}_{\mathbf{k}}=\left(\begin{array}{cccc}
I_{\mathbf{k}}-m_{\mathbf{k}} & t_{1, r} f_{\mathbf{k}}^{*} & t_{2, o} \lambda_{\mathbf{k}}^{*} & t_{1, o} f_{\mathbf{k}}^{*} \\
t_{1, r} f_{\mathbf{k}} & I_{\mathbf{k}}+m_{\mathbf{k}} & t_{1, o} f_{\mathbf{k}} & t_{2, o} \lambda_{\mathbf{k}}^{*} \\
t_{2, o} \lambda_{\mathbf{k}} & t_{1, o} f_{\mathbf{k}}^{*} & I_{\mathbf{k}}+m_{\mathbf{k}} & t_{1, r} f_{\mathbf{k}}^{*} \\
t_{1, o} f_{\mathbf{k}} & t_{2, o} \lambda_{\mathbf{k}} & t_{1, r} f_{\mathbf{k}} & I_{\mathbf{k}}-m_{\mathbf{k}}
\end{array}\right)
$$

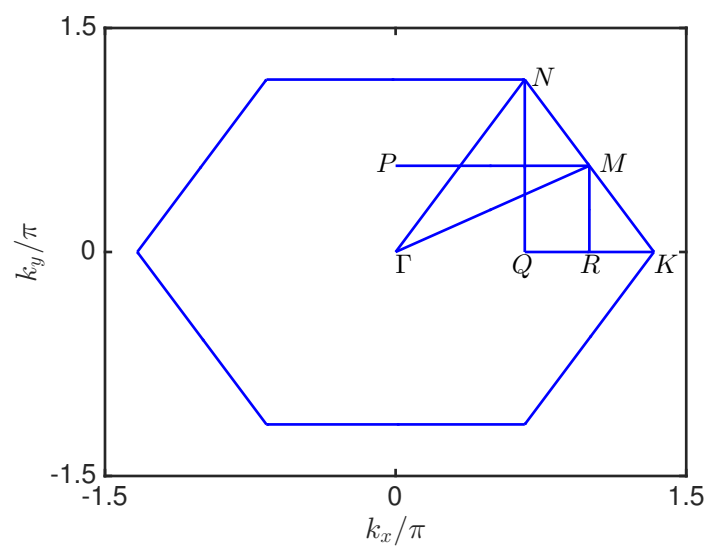

FIG. 6: Color online. The first Brillouin zone of the triangular lattice and the corresponding paths that will be adopted in this section.

where $f_{\mathbf{k}}=1+e^{-i k_{a}}+e^{-i\left(k_{a}+k_{b}\right)}, \quad \lambda_{\mathbf{k}}=2\left[\cos k_{a}+\right.$ $\left.\cos k_{b}+\cos \left(k_{a}+k_{b}\right)\right] ; m_{\mathbf{k}}=2 t_{2} \sin \phi\left[\sin k_{a}+\sin k_{b}-\right.$ $\left.\sin \left(k_{a}+k_{b}\right)\right] ; I_{\mathbf{k}}=3 t_{1, z}+6 t_{2, z}+t_{2} \cos \phi \lambda_{\mathbf{k}}+H \cos \vartheta=$ $3\left(\mathcal{J}_{1}+\mathcal{J}_{2}\right)+t_{2} \cos \phi \lambda_{\mathbf{k}}$. The vectors are $\hat{a}=\sqrt{3} \hat{x}$ and $\hat{b}=-\sqrt{3} \hat{x} / 2+3 \hat{y} / 2$ with $k_{a}=\mathbf{k} \cdot \hat{a}$ and $k_{b}=\mathbf{k} \cdot \hat{b}$. We diagonalize the Hamiltonian numerically via the generalized Bogoluibov transformation $\frac{61}{}$. For $\mathcal{J}_{2} / \mathcal{J}_{1}<1 / 6$ or equivalently $\mathcal{J}_{2} / \mathcal{J}_{1} \rightarrow 0$ the Hamiltonian recovers collinear (canted) Néel antiferromagnet at $H=0\left(H<H_{s}\right)$ as well as collinear ferromagnet at $H=H_{s}$. These limiting cases require the DM interaction for topological features to exist as previously shown 14 19. The dominant $\mathcal{J}_{2}$ limit is different and requires no DM interaction for topological features to exist.

\section{Appendix B: Bilayer triangular-lattice antiferromagnets}

The dominant $\mathcal{J}_{2}$ limit of frustrated honeycomb lattice is isomorphic to the bilayer triangular-lattice Heisenberg antiferromagnets. The conventional Hamiltonian for bilayer triangular-lattice Heisenberg antiferromagnets applicable to real materials is given by

$$
\begin{aligned}
\mathcal{H} & =\mathcal{J}_{2} \sum_{\langle i, j\rangle, \tau}\left[\mathcal{S}_{i \tau}^{\perp} \cdot \mathcal{S}_{j \tau}^{\perp}+\Delta \mathcal{S}_{i \tau}^{z} \mathcal{S}_{j \tau}^{z}\right]-H \sum_{i, \tau} \mathcal{S}_{i, \tau}^{z} \\
& +\mathcal{J}_{1} \sum_{\langle i, j\rangle, \tau \tau^{\prime}}\left[\mathcal{S}_{i \tau}^{\perp} \cdot \mathcal{S}_{j \tau^{\prime}}^{\perp}+\Delta \mathcal{S}_{i \tau}^{z} \mathcal{S}_{j \tau^{\prime}}^{z}\right]
\end{aligned}
$$

where $\tau$ labels the top and bottom layers and $\mathcal{S}_{i}^{\perp}=$ $\left(\mathcal{S}_{i}^{x}, \mathcal{S}_{i}^{y}\right)$. Note that all the interactions are now nearestneighbour $(\mathrm{NN})$. The easy-plane anisotropy lies in the range $0 \leq \Delta \leq 1$. The NN intraplane coupling is $\mathcal{J}_{2}>0$ and the NN interplane coupling is $\mathcal{J}_{1}>0$ with $\mathcal{J}_{1} \ll \mathcal{J}_{2}$, i.e., quasi-2D limit. After the rotation in the spin space 


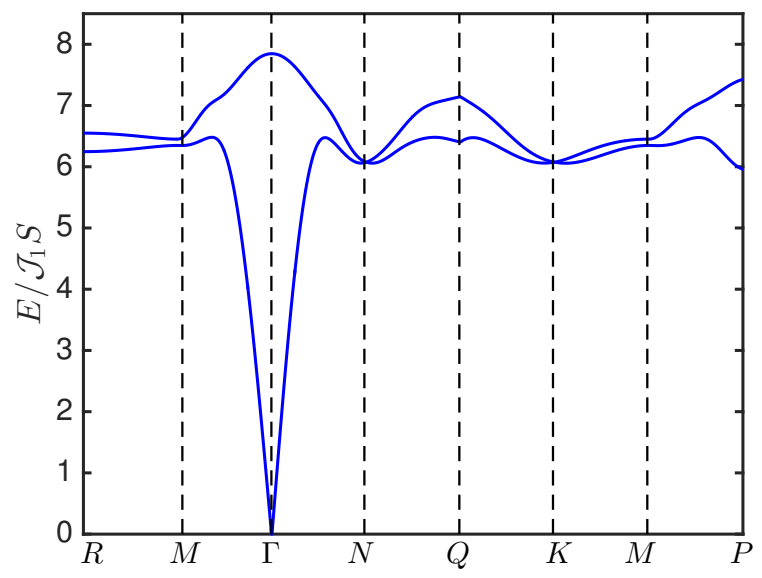

FIG. 7: Color online. Dirac magnon bands (at $N$ and $K$ ) of the bilayer XXZ triangular-lattice antiferromagnet with the conventional $120^{\circ}$ coplanar order at zero magnetic field $H=$ 0 . The plot is generated with $\Delta=0.7$ and $\mathcal{J}_{2} / \mathcal{J}_{1}=1.3$.

(A1) and (A2) we have

$$
\begin{aligned}
\mathcal{H} & =\sum_{i j} \mathcal{J}_{i j}\left[\cos \theta_{i j} \mathcal{S}_{i}^{\prime} \cdot \mathcal{S}_{j}^{\prime}+\sin \theta_{i j} \cos \vartheta \hat{\mathbf{z}} \cdot\left(\mathcal{S}_{i}^{\prime} \times \mathcal{S}_{j}^{\prime}\right)\right. \\
& \left.+\left(\Delta-\cos \theta_{i j}\right)\left(\sin ^{2} \vartheta \mathcal{S}_{i}^{\prime x} \mathcal{S}_{j}^{\prime x}+\cos ^{2} \vartheta \mathcal{S}_{i}^{\prime z} \mathcal{S}_{j}^{\prime z}\right)\right] \\
& -H \cos \vartheta \sum_{i} \mathcal{S}_{i}^{\prime z}
\end{aligned}
$$

where we have retained the free magnon model. $\mathcal{J}_{i j}=$ $\mathcal{J}_{1}\left(\mathcal{J}_{2}\right)$. Because of the antiferromagnetic interplane coupling the spins on the top layer are orientated in the opposite direction to those on the bottom layer, hence $\sin \theta_{i j}=0$ for $\mathcal{J}_{i j}=\mathcal{J}_{1}$ and the scalar-chirality vanishes. However, each layer form a $120^{\circ}$ coplanar order and $\sin \theta_{i j}=\nu_{i j} \sin \left(120^{\circ}\right)$ for $\mathcal{J}_{i j}=\mathcal{J}_{2}$, where $\nu_{i j}= \pm$ for magnon hopping on the top and bottom layers respectively. The scalar-chirality of the non-coplanar structure on both layers are along the positive $z$-axis for $H>0$, whereas for $H<0$ the spins on each triangular-layer flip by $180^{\circ}$. Now $\vartheta \rightarrow \pi+\vartheta$ on each layer. Therefore the net scalar-chirality is nonzero in both cases $\underline{42}$.

We adopt the one-sublattice structure ${ }^{63 \mid 64}$ on each layer of the triangular lattice and label them $A$ and $B$. From figure 1 (a) in the main text, we see that the stacking of the bilayer triangle is such that there are six nearest-neighbours on each layer and three nearest neighbours between the layers. The parameters of the corresponding tight-binding model are

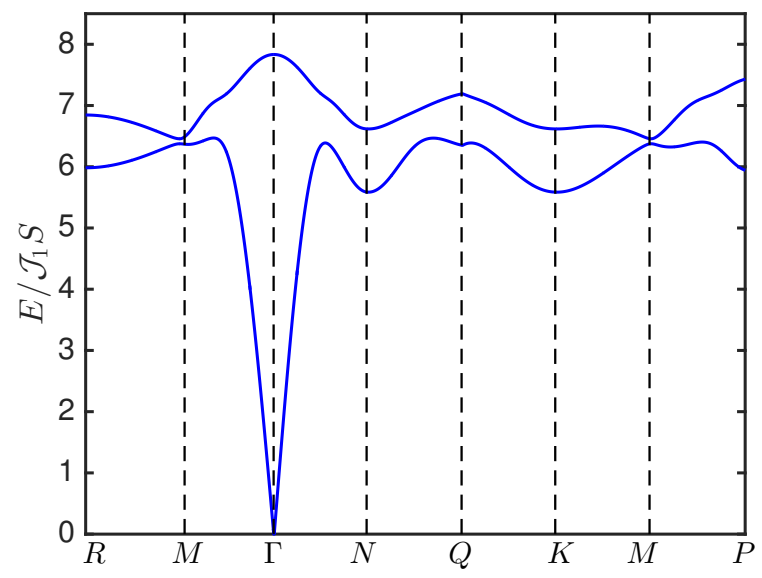

FIG. 8: Color online. Topological magnon bands of the bilayer XXZ triangular-lattice antiferromagnet with the noncoplanar (umbrella) spin configuration at nonzero magnetic field $H=0.2 H_{s}$. The plot is generated with $\Delta=0.7$ and $\mathcal{J}_{2} / \mathcal{J}_{1}=1.3$.

$\left.t_{1, z}=\mathcal{J}_{1}\left[1-(1+\Delta) \cos ^{2} \vartheta\right)\right], t_{1, r}=-\mathcal{J}_{1}\left[1-\frac{1+\Delta}{2} \sin ^{2} \vartheta\right]$

$t_{1, o}=\frac{\mathcal{J}_{1}(1+\Delta)}{2} \sin ^{2} \vartheta, t_{2, z}=\frac{\mathcal{J}_{2}}{2}\left[1-(2 \Delta+1) \cos ^{2} \vartheta\right]$,

$t_{2}=\sqrt{\left(t_{2, r}\right)^{2}+\left(t_{2, m}\right)^{2}}, t_{2, r}=-\frac{\mathcal{J}_{2}}{2}\left(1-\frac{(2 \Delta+1)}{2} \sin ^{2} \vartheta\right)$,

$t_{2, m}=\frac{\sqrt{3} \mathcal{J}_{2}}{2} \cos \vartheta, t_{2, o}=\frac{\mathcal{J}_{2}(2 \Delta+1)}{4} \sin ^{2} \vartheta$,

with $\cos \vartheta=H / H_{s}$ and $H_{s}=3 \mathcal{J}_{1}(1+\Delta)+3 \mathcal{J}_{2}(2 \Delta+1)$.

In the basis $\Psi_{\mathbf{k}}^{\dagger}=\left(a_{\mathbf{k}, A}^{\dagger}, a_{-\mathbf{k}, A}, a_{\mathbf{k}, B}^{\dagger}, a_{-\mathbf{k}, B}\right)$, the momentum space Hamiltonian is given by

$$
\mathcal{H}_{\mathbf{k}}=\left(\begin{array}{cccc}
I_{\mathbf{k}}-m_{\mathbf{k}} & t_{2, o} \lambda_{\mathbf{k}} & t_{1, r} f_{\mathbf{k}}^{*} & t_{1, o} f_{\mathbf{k}}^{*} \\
t_{2, o} \lambda_{\mathbf{k}}^{*} & I_{\mathbf{k}}+m_{\mathbf{k}} & t_{1, o} f_{\mathbf{k}} & t_{1, r} f_{\mathbf{k}} \\
t_{1, r} f_{\mathbf{k}} & t_{1, o} f_{\mathbf{k}}^{*} & I_{\mathbf{k}}+m_{\mathbf{k}} & t_{2, o} \lambda_{\mathbf{k}} \\
t_{1, o} f_{\mathbf{k}} & t_{1, r} f_{\mathbf{k}}^{*} & t_{2, o} \lambda_{\mathbf{k}}^{*} & I_{\mathbf{k}}-m_{\mathbf{k}}
\end{array}\right)
$$

where $f_{\mathbf{k}}=1+e^{-i k_{1}}+e^{-i\left(k_{1}+k_{2}\right)}, \lambda_{\mathbf{k}}=2\left[\cos k_{1}+\cos k_{2}+\right.$ $\left.\cos \left(k_{1}+k_{2}\right)\right] ; m_{\mathbf{k}}=2 t_{2} \sin \phi\left[\sin k_{1}+\sin k_{2}-\sin \left(k_{1}+k_{2}\right)\right] ;$ $I_{\mathbf{k}}=3 t_{1, z}+6 t_{2, z}+t_{2} \cos \phi \lambda_{\mathbf{k}}+H \cos \vartheta=3 \mathcal{J}_{1}+3 \mathcal{J}_{2}+$ $t_{2} \cos \phi \lambda_{\mathbf{k}}$. Here $k_{i}=\mathbf{k} \cdot \hat{e}_{i}$ and the primitive vectors of the triangular lattice are $\hat{e}_{1}=\hat{x}$ and $\hat{e}_{2}=-\hat{x} / 2+\sqrt{3} \hat{y} / 2$. The Brillouin zone paths are depicted in figure (6).

It is evident that when the interplane coupling vanishes, i.e., $\mathcal{J}_{1}=0=t_{1, r}=t_{1, o}$, the Hamiltonian reduces to two decoupled triangular-lattice XXZ antiferromagnets. For $\mathcal{J}_{1} \neq 0$ we have shown the magnon bands of the bilayer triangular-lattice XXZ antiferromagnets at 
$H=0$ and $H=0.2 H_{s}$ respectively in figures. (7) and (8). We see that the magnon bands have the same structure as the frustrated honeycomb lattice shown in the main text. Topological magnon bands in figure (8) directly imply the existence of nonzero Chern numbers, magnon edge modes, and thermal Hall conductivity. We note that $\Delta=1$ has the same topological features as expected.
* Electronic address: sowerre@perimeterinstitute.ca

1 M. Hirschberger et al., Phys. Rev. Lett. 115, 106603 (2015).

${ }^{2}$ R. Chisnell et al., Phys. Rev. Lett. 115, 147201 (2015).

3 Y. Onose et al., Science 329, 297 (2010).

4 T. Ideue et al., Phys. Rev. B. 85, 134411 (2012).

${ }^{5}$ H. Katsura, N. Nagaosa, and P. A. Lee, Phys. Rev. Lett. 104, 066403 (2010).

6 R. Matsumoto and S. Murakami, Phys. Rev. Lett. 106, 197202 (2011). Phys. Rev. B. 84, 184406 (2011).

7 L. Zhang, J. Ren, J. S. Wang, and B. Li, Phys. Rev. B 87, 144101 (2013).

8 A. Mook, J. Henk, and I. Mertig, Phys. Rev. B 90, 024412 (2014). Phys. Rev. B 89, 134409 (2014).

9 R. Shindou et al., Phys. Rev. B 87, 174427 (2013).

10 R. Matsumoto, R. Shindou, and S. Murakami, Phys. Rev. B 89, 054420 (2014).

11 H. Lee, J. H. Han, and P. A. Lee, Phys. Rev. B. 91, 125413 (2015).

12 A. A. Kovalev and V. Zyuzin, Phys. Rev. B 93, 161106(R) (2016).

13 A. Roldán-Molina, A. S. Nunez, and J. Fernández-Rossier, New J. Phys. 18, 045015 (2016).

14 S. A. Owerre, J. Phys.: Condens. Matter 28, 386001 (2016).

15 S. A. Owerre, J. Appl. Phys. 120, 043903 (2016).

16 S. K. Kim et al., Phys. Rev. Lett. 117, 227201 (2016).

17 R. Cheng, S. Okamoto, D. Xiao, Phys. Rev. Lett. 117, 217202 (2016).

18 V. Zyuzin and A. A. Kovalev, Phys. Rev. Lett. 117, 217203 (2016).

19 S. A. Owerre, J. Appl. Phys. 121, 223904 (2017).

20 I. Dzyaloshinsky, J. Phys. Chem. Solids 4, 241 (1958).

21 T. Moriya, Phys. Rev. 120, 91 (1960).

22 F. D. M. Haldane, Phys. Rev. Lett. 61, 2015 (1988).

23 C. L. Kane and E.J. Mele, Phys. Rev. Lett. 95, 226801 (2005).

${ }^{24}$ S. A. Owerre, Phys. Rev. B 95, 014422 (2017).

25 S. A. Owerre, J. Phys.: Cond. Mat. 29, 03LT01 (2017).

26 M. Elhajal, B. Canals, and C. Lacroix, Phys. Rev. B 66, $014422(2002)$.

27 M. Matsuda et al., Phys. Rev. Lett. 105, 187201 (2010).

28 A. Mulder et al., Phys. Rev. B. 81, 214419 (2010).

${ }^{29}$ R. Ganesh et al., Phys. Rev. B. 83, 144414 (2011).

30 J. Oitmaa and R. R. P. Singh, Phys. Rev. B. 85, 014428 (2012).

31 H. Zhang, M. Arlego, and C. A. Lamas, Phys. Rev. B. 89, 024403 (2014).

${ }^{32}$ F. A. Gómez Albarracín and H. D. Rosales, Phys. Rev. B. 92, 144413 (2016).

33 F. Wang, Phys. Rev. B 82, 024419 (2010).
34 Y.-M. Lu and Y. Ran, Phys. Rev. B 84, 024420 (2011).

35 B. K. Clark, D. A. Abanin, and S. L. Sondhi, Phys. Rev. Lett. 107, 087204 (2011).

${ }^{36}$ H. Mosadeq, F. Shahbazi, and S.A. Jafari, J. Phys. Condens. Matter 23, 226006 (2011).

37 A. F. Albuquerque et al., Phys. Rev. B 84, 024406 (2011).

38 R. Bishop et al., J. Phys. Condens. Matter 24, 236002 (2012).

39 F. Mezzacapo and M. Boninsegni, Phys. Rev. B 85, 060402 (2012).

40 R. Ganesh, J. van den Brink, and S. Nishimoto Phys. Rev. Lett. 110, 127203 (2013).

41 K. Jiang et al., Phys. Rev. Lett. 114, 216402 (2015).

42 D. Grohol et al., Nat. Mater. 4, 323 (2005).

43 Y. Doi, Y. Hinatsu, and K. Ohoyama, J. Phys. Condens. Matter 16, 8923 (2004).

44 Y. Shirata et al., Phys. Rev. Lett. 108, 057205 (2012).

${ }^{45}$ H. D. Zhou et al., Phys. Rev. Lett. 109, 267206 (2012).

46 T. Susuki et al., Phys. Rev. Lett. 110, 267201 (2013).

47 G. Quirion et al., Phys. Rev. B 92, 014414 (2015).

48 P. A. Maksimov, M. E. Zhitomirsky, and A. L. Chernyshev, Phys. Rev. B 94, 140407(R) (2016).

49 J. Ma et al., Phys. Rev. Lett. 116, 087201 (2016).

${ }^{50}$ K. Hirakawa, H. Kadowaki, and K. Ubukoshi, J. Phys. Soc. Jpn. 52, 1814 (1983).

51 H. Kadowaki, K. Ubukoshi, and K. Hirakawa, J. Phys. Soc. Jpn., 54, 363 (1985).

52 M. F. Collins and O. A. Petrenko, Can. J. Phys. 75605 (1997)

53 H. Takatsu et al., Phys. Rev. Lett. 105, 137201 (2010).

54 J. Romhányi, K. Penc, and R. Ganesh, Nat. Commun. 6, 6805 (2015).

55 M. Malki and K. P. Schmidt, Phys. Rev. B 95, 195137 (2017).

56 P. Laurell, G. A. Fiete, Phys. Rev. Lett. 118, 177201 (2017).

57 P. A. McClarty et al., Nat. Phys., doi:10.1038/nphys4117 (2017).

58 S. Okubo et al., Phys. Rev. B 86, 140401(R) (2012).

59 Y. Singh and P. Gegenwart, Phys. Rev. B 82, 064412 (2010).

60 D. Watanabe et al., Proc. Natl. Acad. Sci. USA 113, 8653 (2016).

61 J. -P. Blaizot and G. Ripka, Quantum Theory of Finite Systems (MIT Press, Cambridge, MA, 1986).

62 T. Holstein and H. Primakoff, Phys. Rev. 58, 1098 (1940).

63 A. V. Chubukov et al., J. Phys.: Condens. Matter 6, 8891 (1994).

64 S. Sachdev, Phys. Rev. B 45, 12377 (1992). 